\title{
Single-Shot X-Ray Phase-Contrast Computed Tomography with Nonmicrofocal Laboratory Sources
}

\author{
P. C. Diemoz, ${ }^{1,{ }^{*}}$ C. K. Hagen, ${ }^{1}$ M. Endrizzi, ${ }^{1}$ M. Minuti, ${ }^{2,3}$ R. Bellazzini, ${ }^{2,3}$ L. Urbani, ${ }^{4}$ P. De Coppi, ${ }^{4}$ and A. Olivo ${ }^{1}$ \\ ${ }^{1}$ Department of Medical Physics and Biomedical Engineering, UCL, WC1E 6BT London, United Kingdom \\ ${ }^{2}$ PIXIRAD Imaging Counters s.r.l., clo INFN Pisa, Largo B. Pontecorvo 3, 56127 Pisa, Italy \\ ${ }^{3}$ INFN, Pisa Section, Largo B. Pontecorvo 3, 56127 Pisa, Italy \\ ${ }^{4}$ UCL, Institute of Child Health and Great Ormond Street Hospital, London WC1N IEH, United Kingdom \\ (Received 16 August 2016; revised manuscript received 10 March 2017; published 28 April 2017)
}

\begin{abstract}
We present a method that enables performing x-ray phase-contrast imaging (XPCI) computed tomography with a laboratory setup using a single image per projection angle, eliminating the need to move optical elements during acquisition. Theoretical derivation of the method is presented, and its validity conditions are provided. The object is assumed to be quasihomogeneous, i.e., to feature a ratio between the refractive index and the linear attenuation coefficient that is approximately constant across the field of view. The method is experimentally demonstrated on a plastics phantom and on biological samples using a continuous rotation acquisition scheme achieving scan times of a few minutes. Moreover, we show that such acquisition times can be further reduced with the use of a high-efficiency photon-counting detector. Thanks to its ability to substantially simplify the image-acquisition procedure and greatly reduce collection times, we believe this method represents a very important step towards the application of XPCI to real-world problems.
\end{abstract}

DOI: 10.1103/PhysRevApplied.7.044029

\section{INTRODUCTION}

$\mathrm{X}$-ray phase-contrast imaging (XPCI) techniques enable the achievement of highly improved contrast compared to conventional absorption-based imaging methods, thanks to their ability to detect the $\mathrm{x}$-ray phase shift undergone by the beam upon passing through the sample in addition to $\mathrm{x}$-ray attenuation [1]. This has led to increasing interest and significant research efforts dedicated to this topic, in particular, research on translating XPCI from synchrotron radiation facilities to tabletop setups making use of conventional x-ray tubes [2-4].

Despite the high expectations, however, the implementation of XPCI in normal research laboratories, as well as in industrial and clinical facilities, remains limited. One of the main limitations is represented by the excessively long acquisition times often required, especially for computed tomography (CT) applications. Long exposure times are due, in particular, to the need to use an x-ray source with a very small (approximately microns) focus (thus, severely limiting the available flux) [2] and/or the need to acquire several images as input for the phase retrieval at different positions of the optical elements [3,5]. Not only does the

\footnotetext{
* Corresponding author. p.diemoz@ucl.ac.uk

Published by the American Physical Society under the terms of the Creative Commons Attribution 4.0 International license. Further distribution of this work must maintain attribution to the author(s) and the published article's title, journal citation, and DOI.
}

latter requirement increase the total exposure time, but it also substantially complicates the $\mathrm{CT}$ acquisition procedure and introduces significant dead times. In fact, in typical XPCI CT implementations, the movement of the optical elements and the acquisition of several frames is performed at every rotation angle before moving to the next angle and repeating this sequence [6,7]. This acquisition scheme is normally preferred to the one where a whole sample rotation is carried out before changing the optical elements position because of stability reasons (i.e., to maximize consistency between frames acquired at the same angle). However, it introduces considerable dead times and prevents the use of continuous rotation schemes, which can considerably speed up the acquisition. An exception is represented by the so-called "reverse-projection" method, which has been applied both with synchrotron [8-9] and laboratory setups [10]. However, this method relies on strict alignment of the optical elements and, effectively, requires the acquisition of twice as many angles than used in the CT reconstruction. Other methods have been developed that do not need multiple images per projection angle; however, they are based on XPCI setups that require the use of either synchrotron radiation [11-12], microfocal sources [13-14], or strong energy filtration of the radiation emitted by a conventional x-ray tube [15], again resulting in long exposure times.

In this article, we present a method that offers a practical solution to the above-mentioned problems by requiring the acquisition of only one image per angular view and enabling the use of continuous rotation acquisition schemes. The method is based on the so-called edge-illumination (EI) technique, which was implemented with synchrotron 
radiation [16-17] and then demonstrated to be compatible with the use of polychromatic and divergent radiation emitted by laboratory nonmicrofocal x-ray tubes [4-5,18-19]. This latter property derives directly from the intrinsically incoherent nature of the EI technique, which, in fact, can be accurately described using pure geometrical optics [20-22].

\section{THEORY}

The EI setup makes use of two absorbing masks made of alternating absorbing and transmitting lines: one mask is placed just before the sample, while the other is in front of the detector [see Fig. 1(a)]. The masks are slightly misaligned with respect to each other so that a fraction of the photons exiting from the first mask passes through the apertures of the second, while the remaining fraction strikes the absorbing septa and will, thus, be stopped. Each of the apertures in the detector mask is aligned with a line of pixels on the detector, which can then count the transmitted intensity. In this configuration, a slight change in the beamlet's direction caused by refraction from the object leads to a change in the proportion of photons being stopped by the second mask and, thus, to a change in the signal on the detector. The masks, therefore, have the effect of converting the sample refraction into a measurable intensity modulation on the detector.

For every monochromatic component of wavelength $\lambda$ in the beam, the refraction angle in the direction $x$ orthogonal to the mask lines is equal to $\Delta \theta_{x}(x, y ; \lambda)=$ $k^{-1} \nabla_{x} \phi(x, y ; \lambda)$, where $k=2 \pi / \lambda$ is the wave number, $\phi(x, y ; \lambda)=k \int d z \delta(x, y, z ; \lambda)$ is the phase shift, $\delta$ is the object refractive index, $z$ is the beam axis, and $\nabla_{x}$ indicates differentiation along $x$. Transmission through the object is equal to $T(x, y ; \lambda)=\exp [-p(x, y ; \lambda)]=\exp \left[-\int d z \mu(x, y\right.$, $z ; \lambda)]$, where $\mu$ is the object linear attenuation coefficient. The signal on the detector can then be described as [17-18]

$S(x, y ; \lambda)=N T(x, y ; \lambda) C\left[x_{e}-k^{-1} z_{2} \nabla_{x} \phi(x, y ; \lambda) ; \lambda\right]$,

where $N$ represents the number of photons per pixel exiting the first mask, and $C\left(x_{e} ; \lambda\right)$ is the fraction of those photons being transmitted through the second mask as a function of the misalignment $x_{e}$ between the two masks. $C\left(x_{e} ; \lambda\right)$ is usually referred to as the "illumination curve," and its values range from approximately 1 (for aligned masks) to approximately 0 (for totally misaligned masks) [see Fig. 1(b)]. The effect of the sample is to attenuate the beam intensity and, due to refraction, to shift the argument of the illumination curve by $-\Delta x=-k^{-1} z_{2} \nabla_{x} \phi$, where $z_{2}$ is the propagation distance between the sample and the detector mask, and $\Delta x$ is the spatial shift of the beam along $x$. The refraction signal is to first approximation proportional to the phase derivative. In fact, if an image is acquired on one of the slopes of the illumination curve, and if the beam shift $\Delta x$ is sufficiently small, a linear approximation of the illumination curve can be safely used, giving $S(x, y ; \lambda)=$ $N T(x, y ; \lambda)\left[C\left(x_{e} ; \lambda\right)-k^{-1} z_{2} \nabla_{x} \phi(x, y ; \lambda) C^{\prime}\left(x_{e} ; \lambda\right)\right]$, where $C^{\prime}\left(x_{e} ; \lambda\right)=\partial C\left(x_{e} ; \lambda\right) / \partial x_{e}$ is the first derivative of the illumination curve.

Equation (1) represents the EI signal along the direction orthogonal to the mask lines. It can be shown that in the direction $y$ parallel to the mask lines, the signal is the same as the one that is obtained without masks [23]. In the approximation of the near-field regime, this signal can be expressed by using the well-known transport-of-intensity equation [24-25] (note that, in the direction orthogonal to the apertures, the same signal is effectively converted into an EI signal due to the presence of both sample and detector masks [21]). By adding this term along $y$ to the expression for the signal, as well as the convolution with the detector response function, one obtains

$$
\begin{aligned}
S_{n}(x, y ; \lambda)= & \{T(x, y ; \lambda)-T(x, y ; \lambda) \\
& \times \frac{C^{\prime}\left(x_{e} ; \lambda\right)}{C\left(x_{e} ; \lambda\right)} k^{-1} z_{2} \nabla_{x} \phi(x, y ; \lambda) \\
& \left.-k^{-1} z_{2} \nabla_{y}\left[T \nabla_{y} \phi(x, y ; \lambda)\right]\right\} * \operatorname{LSF}_{y}(y),
\end{aligned}
$$

where $S_{n}=S /(N C)$ is the normalized signal, $\operatorname{LSF}(y)$ is the line-spread function of the detector along $y$, and $*$ indicates one-dimensional (1D) convolution. In Eq. (2), the first term is the conventional attenuation signal, the second term is the EI signal along $x$, and the third term the

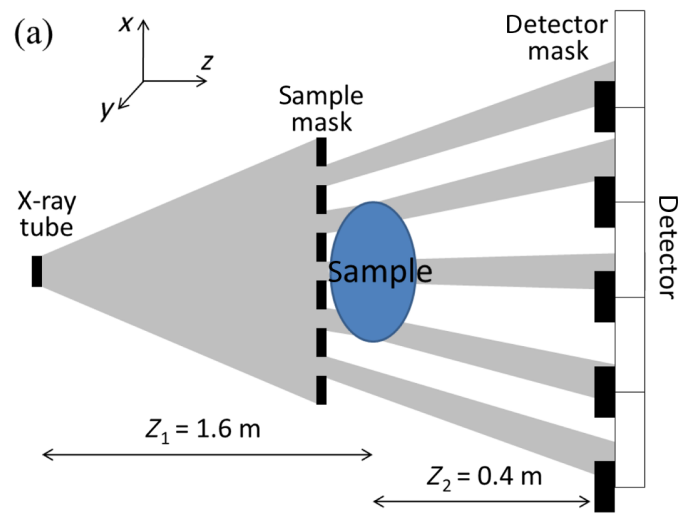

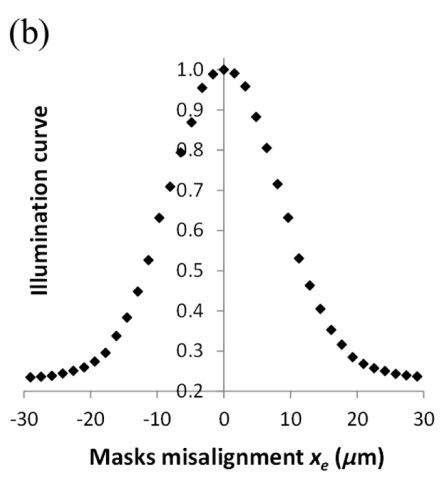

FIG. 1. (a) Schematic diagram of the EI table-top setup (not to scale). (b) Example of illumination curve measured experimentally in our laboratory setup and normalized to 1 . 
free-space-propagation (FSP) signal along $y$. In general, the signal recorded by the EI setup contains a mixture of attenuation and refraction contrast, as seen from Eq. (2). Therefore, two different images are usually needed to separate and quantify these two quantities: these images are usually acquired at the left and right slopes of the illumination curve and then mathematically combined [17-18]. Following a recent publication, however, we assume here that the sample is quasihomogeneous, i.e., that the ratio $\gamma(\lambda)=\delta(x, y, z ; \lambda) /$ $\mu(x, y, z ; \lambda)$ is approximately constant throughout the field of view [26]. This approximation has been used extensively in the framework of the FSP technique [13] and was shown to hold in several practical cases, in particular for biological soft tissues. Under this assumption, the phase and attenuation reduce to a single unknown as $\phi=-k \gamma \int d t \mu(t)$, thus, requiring only a single image for the retrieval. In the following, we further assume that the illumination curve $C$ is independent of energy. This is demonstrated experimentally in Ref. [27] and is true if (1) the masks are sufficiently thick to absorb all the $\mathrm{x}$ rays incident on the absorbing septa, and (2) the projected source size is sufficiently large so as to wash out diffraction peaks [18]. By exploiting the equality $\exp (-p) \nabla_{x, y} p=-\nabla_{x, y} \exp (-p)$, Eq. (2) can be written for a single image acquired on one of the slopes of the illumination function as [26]

$$
\begin{aligned}
S_{n}(\lambda)= & {\left[\mathrm{LSF}_{y} *-\gamma(\lambda) J_{\mathrm{EI}} \mathrm{LSF}_{y} * \nabla_{x}\right.} \\
& \left.-\gamma(\lambda) z_{2} \mathrm{LSF}_{y} * \nabla_{y}^{2}\right] \exp [-p(\lambda)],
\end{aligned}
$$

where for simplicity of notation, we have discarded the dependences upon $x$ and $y$ and where we have defined $J_{\mathrm{EI}}=z_{2} C^{\prime}\left(x_{e}\right) / C\left(x_{e}\right)$. By taking the 2D Fourier transform of both sides of Eq. (3), we obtain

$$
\begin{aligned}
F\left\{S_{n}(\lambda)\right\}= & {\left[1-2 \pi i \gamma(\lambda) J_{\mathrm{EI}} f_{x}+4 \pi^{2} \gamma(\lambda) z_{2} f_{y}^{2}\right] \operatorname{MTF}_{y}\left(f_{y}\right) } \\
& \times F\{\exp [-p(\lambda)]\},
\end{aligned}
$$

where $\operatorname{MTF}_{y}\left(f_{y}\right)=F\left\{\operatorname{LSF}_{y}(y)\right\}$ is the modulation transfer function of the detector in the direction parallel to the mask lines, and $f_{x}$ and $f_{y}$ are the spatial frequencies along $x$ and $y$. Equation (4) establishes a simple relationship between the measured image and the unknown quantity $p(x, y ; \lambda)$, which can be directly inverted in the monochromatic case to obtain the latter quantity. With a polychromatic beam, however, the measured detector signal is the weighted sum of all monochromatic components, i.e., $S_{n}(x, y)=\int d \lambda f(\lambda) S_{n}(x, y ; \lambda)$, where the weight $f(\lambda)$ includes both the source spectrum and the energy response of the detector. An equation equivalent to Eq. (4) can then be written in the polychromatic case as

$F\left\{S_{n}\right\}=\left(1-i 2 \pi f_{x} J_{\mathrm{EI}} \gamma_{\mathrm{ef} f}+4 \pi^{2} f_{y}^{2} z_{2} \gamma_{\mathrm{eff}}\right) \mathrm{MTF}_{y}\left(f_{y}\right) F\left\{T_{\text {eff }}\right\}$,

where we have defined the effective transmission $T_{\text {eff }}(x, y) \equiv$ $\int d \lambda f(\lambda) \exp [-p(x, y ; \lambda)]$ and the effective $\delta / \mu$ ratio $\gamma_{\text {eff }} \equiv$ $T_{\text {eff }}^{-1}\left\{\int_{\lambda_{\min }}^{\lambda_{\max }} d \lambda f(\lambda) \gamma(\lambda) \exp [-p(\lambda)]\right\}$. Moreover, we have assumed that $\gamma_{\text {eff }}$ is approximately constant within the field of view so that it can be taken out of the Fourier transform. It can be shown that this amounts to assuming that no significant beam hardening occurs as an effect of broad polychromaticity and object attenuation, such that the spectrum does not vary significantly among different regions of the image. Equation (5) can then be easily inverted to obtain the effective object absorption map:

$$
\int d z \mu_{\mathrm{eff}}(x, y, z)=-\log \left[F^{-1}\left\{\frac{F\left\{S_{n}(x, y)\right\}}{\operatorname{MTF}_{y}\left(f_{y}\right)\left(1-i 2 \pi J_{\mathrm{EI}} \gamma_{\mathrm{eff}} f_{x}+4 \pi^{2} z_{2} \gamma_{\mathrm{eff}} f_{y}^{2}\right)}\right\}\right]
$$

The 3D distributions of $\mu_{\text {eff }}$ and $\delta_{\text {eff }}=\gamma_{\text {eff }} \mu_{\text {eff }}$ can then be calculated by applying standard CT reconstruction methods such as filtered backprojection (FBP) from a single image $S_{n}(x, y)$ acquired on one of the slopes of the illumination curve. It must be noted that beam-hardening artifacts might affect the quality of the CT reconstruction, especially for large and/or dense samples, similar to conventional absorption-based CT [28]; therefore, adaptation to XPCI of the correction methods developed for conventional CT might be necessary to correct for these effects.

\section{EXPERIMENTAL VERIFICATION}

The proposed single-image method is validated experimentally using one of the EI laboratory setups installed at University College London. The setup is based on a Rigaku
MicroMax $007 \mathrm{HF}$ x-ray tube with a molybdenum rotating target. The x-ray tube is operated at $40 \mathrm{kV}$ and $25 \mathrm{~mA}$ and has an effective focal spot of about $70 \mu \mathrm{m}$ (full width at half maximum). The detector is a Hamamatsu C9732DK flat panel, a passive-pixel CMOS with a pixel size of $50 \times 50 \mu \mathrm{m}^{2}$. The sample and detector masks are manufactured by gold electroplating on a graphite substrate (Creatv Microtech, Inc., Potomac, MD). They are oriented with their apertures in the vertical direction and are placed, respectively, 1.60 and $1.98 \mathrm{~m}$ from the source. The pitch and aperture size of the masks are, respectively, 79 and $10 \mu \mathrm{m}$ (sample mask) and 98 and $17 \mu \mathrm{m}$ (detector mask). The setup employs a lineskipping configuration, whereby only every other detector column is illuminated in order to reduce cross talk between pixels. It is worth mentioning that the spatial sampling in the 

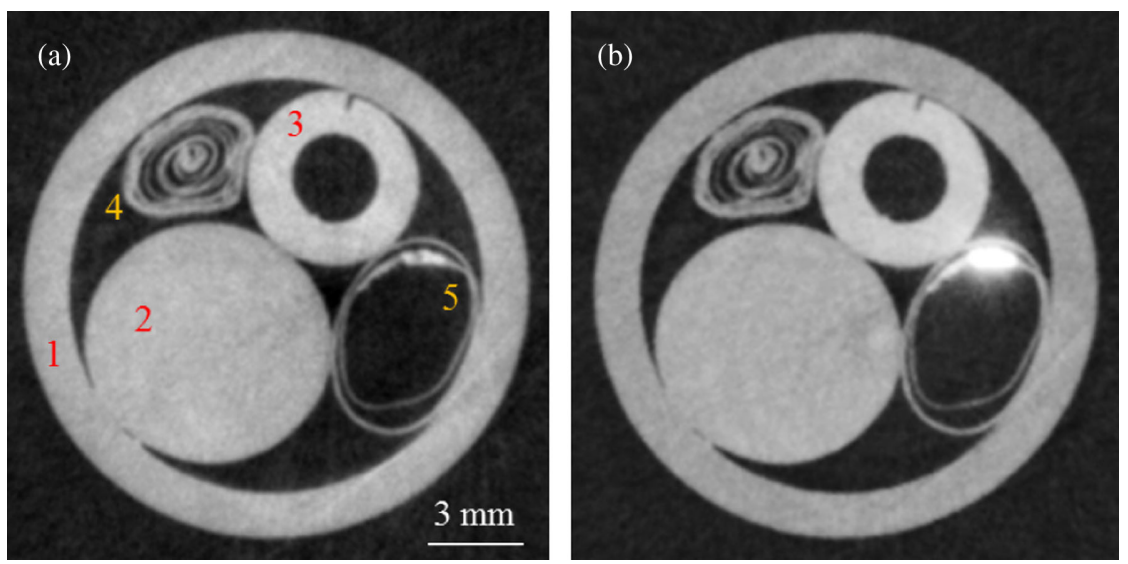

FIG. 2. Reconstructed axial slices of the plastic phantom obtained (a) with the conventional EI retrieval algorithm and (b) with the single-image-retrieval algorithm. The objects inside the phantom are (1) a hollow plastic cylinder, (2) a plastic rod, (3) a second, smaller hollow plastic cylinder, (4) rolled plastic paraffin film, and (5) chalk inside a plastic container. object plane along the horizontal direction is equal to the sample mask pitch, i.e., $79 \mu \mathrm{m}$ in this case, unless an additional scan of the sample is performed in the direction orthogonal to the mask lines [10].

In the first example, we compare the new single-image method with the conventional EI retrieval on a custom-built phantom consisting of (1) a hollow plastic cylinder, (2) a plastic rod, (3) a second, smaller hollow plastic cylinder, (4) rolled plastic paraffin film, and (5) chalk inside a plastic container [cf. Fig. 2(a)]. The single-image acquisition is performed on the left slope of the illumination curve. A continuous rotation of the object over $360^{\circ}$ is carried out with an angular step of $0.9^{\circ}$ (corresponding to a total of 400 projections) and an exposure time of $1 \mathrm{~s}$ per projection. The total acquisition time is, therefore, equal to $6 \mathrm{~min} 40 \mathrm{~s}$. For the conventional EI retrieval method, the same acquisition is repeated at the right slope of the illumination curve, leading to a total time of just above $13 \mathrm{~min} 20 \mathrm{~s}$. Twenty images without object (flat fields) are acquired at both positions to correct for beam, masks, and detector nonuniformities. Equation (6) followed by the FBP algorithm with a ramp filter is used for CT reconstruction of single-image data. The exact materials of the sample are unknown, but an average value of $\gamma_{\text {eff }}=9.32 \times 10^{-7} \mathrm{~cm}$ is assumed, corresponding to $\delta=6.62 \times 10^{-7}$ and $\beta=0.71 \mathrm{~cm}^{-1}$ for polyethylene terephthalate at 21 and $19 \mathrm{keV}$, respectively (approximate effective energies for the refraction and attenuation signals [7]). For the standard EI reconstruction, the refraction angle map is first extracted for every angle using the two-imageretrieval method presented in Refs. [17-18] and the 3D map of the refractive index reconstructed using the FBP algorithm with the Hilbert filter [7].

Axial slices of the sample obtained using the two-image and single-image algorithms are presented in Figs. 2(a) and 2(b), respectively. The image reconstructed with the proposed single-image algorithm shows comparable image quality and qualitative agreement with the other reconstruction. Only the region surrounding the chalk is affected by blurring artifacts due to a much smaller value of $\gamma_{\text {eff }}$ for this material $\left(\gamma_{\text {eff }}=7.1 \times 10^{-8}\right.$ for calcite). This unwanted effect was already observed in single-distance retrieval methods based on the FSP technique, and algorithms aimed to eliminate it were developed [29-30]. The average reconstructed values for $\delta$ are for the two-image and single-image algorithms, respectively, big hollow cylinder $\left(5.8 \times 10^{-7}, 5.1 \times 10^{-7},-12 \%\right.$ difference), $\operatorname{rod}\left(6.2 \times 10^{-7}, 5.7 \times 10^{-7},-7.4 \%\right.$ difference $)$, and small hollow cylinder $\left(6.7 \times 10^{-7}, 7.1 \times 10^{-7},+5.7 \%\right.$ difference). As can be seen, the reconstructed values obtained with the proposed method show reasonable agreement with the conventional EI retrieval, despite the exact ratio $\gamma_{\text {eff }}$ of the considered materials being unknown and not the same for the various materials. It should be mentioned that, due to the divergent nature of the beam, a fan-beam CT reconstruction [31] will be potentially more accurate than the one used here based on the assumption of a parallel beam. However, for the small sample sizes considered in this study, this was not considered to be important (i.e., the reconstructed images do not show blurring or distortion effects attributable to the fan beam). Fan-beam CT reconstruction will need to be implemented in the future when the proposed method will be scaled up for use on larger samples.

In the second example, we show that the method can provide high-quality reconstructions also for biological objects with complex geometry, despite the simplified setup and the short acquisition times. In Figs. 3(a)-3(d), in particular, we show reconstructed axial slices and sagittal slices for two different samples: a ground beetle [Figs. 3(a) and 3(b)] and a freeze-dried rat heart [Figs. 3(c) and 3(d)]. Acquisitions are performed using the same parameters as for the plastic phantom with the same total acquisition time of $6 \mathrm{~min} 40 \mathrm{~s}$. In this case, the precise chemical composition of the samples is unknown, and it is, thus, chosen to adjust the value of $\gamma_{\text {eff }}$ in Eq. (6) until an optimal image is reconstructed from a qualitative point of view (as is common procedure also for the single-image methods based on FSP [13]). The reconstructions show that the sample structures can be clearly visualized and, thus, that the proposed method is very interesting also for the imaging of biological samples.

Finally, we present preliminary data showing that the results obtained so far can be still considerably improved 

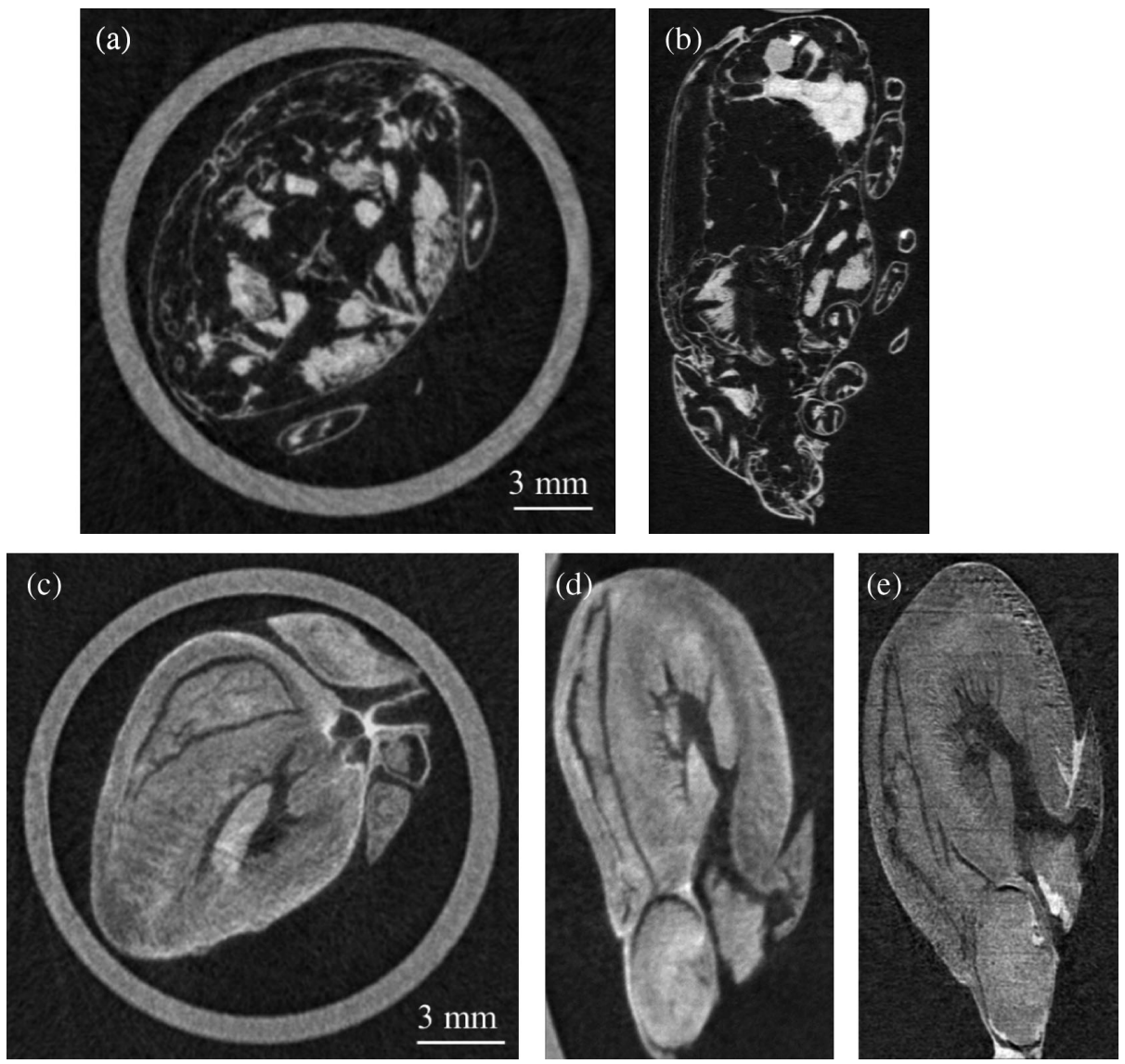

FIG. 3. Ground beetle: (a) axial slice reconstructed using the single-image method and (b) a vertical section through the volume. Rat heart: (c) axial slice reconstructed using the single-image method, (d) a vertical section through the volume, (e) corresponding preliminary result obtained using the single-photon-counting Pixirad detector.

with further optimizations of the setup. In particular, we have repeated the $\mathrm{CT}$ of the rat heart by replacing the Hamamatsu CMOS detector with Pixirad, a directconversion single-photon-counting detector [32]. The virtually negligible electronic noise and the almost $100 \%$ detection efficiency enable us to reduce the scan times without significantly affecting the image quality. In Fig. 3(e), we show the reconstruction obtained with a total acquisition time of only $3 \mathrm{~min} 20 \mathrm{~s}$ (400 projections, $0.5-\mathrm{s}$ exposure, continuous rotation) in a similar position as to that depicted in Fig. 3(d). Importantly, we see that the image quality is not affected due to the shorter integration time, and, instead, a slightly improved spatial resolution is observed due to the lower cross talk between pixels. It should also be pointed out that, since cross talk is smaller, a line-skipping configuration would not be needed with this detector. Masks with a period reduced by half have already been designed for this setup and are expected to provide even better image quality and/or enable shorter exposure times when they become available.

\section{CONCLUSION}

We demonstrate that the proposed single-image XPCI $\mathrm{CT}$ method enables achieving acquisition times well below those previously obtained with XPCI setups based on standard X-ray tubes. To our knowledge, in particular, the shortest acquisition time reported in the literature was 25 min (obtained by our group using a laboratory setup based on EI [10]), while most other studies were conducted using acquisition times on the order of few to several hours [6,33-34]. Because of the simplicity of the setup, which eliminates the need for a movement of the optical elements during acquisition, and to the very short acquisition times it enables achieving, we expect the proposed method to find application in a variety of fields where fast acquisitions are of paramount importance.

\section{ACKNOWLEDGMENTS}

This work is supported by the UK Engineering and Physical Sciences Research Council (Grant No. EP/ I021884/1). P. C. D. is supported by Marie Curie Career Integration Grant No. PCIG12-GA-2012-333990 within the Seventh Framework Programme of the European Union. M. E. is supported by the Royal Academy of Engineering under the Research Fellowship scheme.

[1] S. W. Wilkins, Y. I. Nesterets, T. E. Gureyev, S. C. Mayo, A. Pogany, and A.W. Stevenson, On the evolution and relative merits of hard X-ray phase-contrast imaging methods, Phil. Trans. R. Soc. A 372, 20130021 (2014). 
[2] S. W. Wilkins, T. E. Gureyev, D. Gao, A. Pogany, and A. W. Stevenson, Phase-contrast imaging using polychromatic hard X-rays, Nature (London) 384, 335 (1996).

[3] F. Pfeiffer, T. Weitkamp, O. Bunk, and C. David, Phase retrieval and differential phase-contrast imaging with lowbrilliance X-ray sources, Nat. Phys. 2, 258 (2006).

[4] A. Olivo and R. Speller, A coded-aperture technique allowing $\mathrm{X}$-ray phase contrast imaging with conventional sources, Appl. Phys. Lett. 91, 074106 (2007).

[5] P. R. T. Munro, K. Ignatyev, R. D. Speller, and A. Olivo, Phase and absorption retrieval using incoherent $\mathrm{X}$-ray sources, Proc. Natl. Acad. Sci. U.S.A. 109, 13922 (2012).

[6] S. Grandl, M. Willner, J. Herzen, D. Mayr, S. D. Auweter, A. Hipp, F. Pfeiffer, M. Reiser, and K. Hellerhoff, Evaluation of phase-contrast CT of breast tissue at conventional X-ray sources-Presentation of selected findings, Z. Med. Phys. 23, 212 (2013).

[7] C. K. Hagen, P. R. T. Munro, M. Endrizzi, P. C. Diemoz, and A. Olivo, Low-dose phase contrast tomography with conventional X-ray sources, Med. Phys. 41, 070701 (2014).

[8] M. Wang, P. P. Zhu, K. Zhang, X. F. Hu, W. X. Huang, Y. W. Cen, Q. X. Yuan, X. L. Yu, and J. Y. Wang, A new method to extract angle of refraction in diffraction enhanced imaging computed tomography, J. Phys. D 40, 6917 (2007).

[9] P. Zhu, K. Zhang, Z. Wang, Y. Liu, X. Liu, Z. Wu, S. A. McDonald, F. Marone, and M. Stampanoni, Low-dose, simple, and fast grating-based x-ray phase-contrast imaging, Proc. Natl. Acad. Sci. U.S.A. 107, 13576 (2010).

[10] C. K. Hagen, M. Endrizzi, P. C. Diemoz, and A. Olivo, Reverse projection retrieval in edge illumination $\mathrm{x}$-ray phase contrast computed tomography, J. Phys. D 49, 255501 (2016).

[11] D. Briedis, K. K. W. Siu, D. M. Paganin, K. M. Pavlov, and R. A. Lewis, Analyser-based mammography using singleimage reconstruction, Phys. Med. Biol. 50, 3599 (2005).

[12] K. S. Morgan, D. M. Paganin, and K. K. W. Siu, X-ray phase imaging with a paper analyzer, Appl. Phys. Lett. 100, 124102 (2012).

[13] D. Paganin, S. C. Mayo, T. E. Gureyev, P. R. Miller, and S. W. Wilkins, Simultaneous phase and amplitude extraction from a single defocused image of a homogeneous object, J. Microsc. 206, 33 (2002).

[14] B. D. Arhatari, G. van Riessen, and A. Peele, Polychromatic $\mathrm{X}$-ray tomography: Direct quantitative phase reconstruction, Opt. Express 20, 23361 (2012).

[15] D. J. Vine, D. M. Paganin, K. M. Pavlov, J. Kräußlich, O. Wehrhan, I. Uschmann, and E. Förster, Analyzer-based phase contrast imaging and phase retrieval using a rotating anode X-ray source, Appl. Phys. Lett. 91, 254110 (2007).

[16] A. Olivo, F. Arfelli, G. Cantatore, R. Longo, R. H. Menk, S. Pani, M. Prest, P. Poropat, and L. Rigon, G. Tromba, E. Vallazza, and E. Castelli, An innovative digital imaging setup allowing a low-dose approach to phase contrast applications in the medical field, Med. Phys. 28, 1610 (2001).

[17] P. C. Diemoz, M. Endrizzi, C. E. Zapata, Z. D. Pešić, C. Rau, A. Bravin, I. K. Robinson, and A. Olivo, X-Ray PhaseContrast Imaging with Nanoradian Angular Resolution, Phys. Rev. Lett. 110, 138105 (2013).

[18] P. C. Diemoz, C. K. Hagen, M. Endrizzi, and A. Olivo, Sensitivity of laboratory based implementations of edge illumination x-ray phase contrast imaging, Appl. Phys. Lett. 103, 244104 (2013).

[19] A. Olivo, S. Gkoumas, M. Endrizzi, C. K. Hagen, M. B. Szafraniec, P. C. Diemoz, P. R. T. Munro, K. Ignatyev, B. Johnson, J. A. Horrocks, S. J. Vinnicombe, J. L. Jones, and R. D. Speller, Low-dose phase contrast mammography with conventional x-ray sources, Med. Phys. 40, 090701 (2013).

[20] P. R. T. Munro, K. Ignatyev, R. D. Speller, and A. Olivo, The relationship between wave and geometrical optics models of coded aperture type x-ray phase contrast imaging systems, Opt. Express 18, 4103 (2010).

[21] P. C. Diemoz and A. Olivo, On the origin of contrast in edge illumination X-ray phase-contrast imaging, Opt. Express 22, 28199 (2014).

[22] M. Endrizzi, P. C. Diemoz, T. P. Millard, J. L. Jones, R. D. Speller, I. K. Robinson, and A. Olivo, Hard X-ray dark-field imaging with incoherent sample illumination, Appl. Phys. Lett. 104, 024106 (2014).

[23] P. C. Diemoz, F. A. Vittoria, and A. Olivo, Spatial resolution of edge illumination x-ray phase-contrast imaging, Opt. Express 22, 15514 (2014).

[24] M. R. Teague, Deterministic phase retrieval: A Green's function solution, J. Opt. Soc. Am. 73, 1434 (1983).

[25] T. E. Gureyev, Y. I. Nesterets, A. W. Stevenson, P. R. Miller, A. Pogany, and S.W. Wilkins, Some simple rules for contrast, signal-to-noise and resolution in in-line $\mathrm{x}$-ray phase-contrast imaging, Opt. Express 16, 3223 (2008).

[26] P. C. Diemoz, F. A. Vittoria, C. K. Hagen, M. Endrizzi, P. Coan, E. Brun, U. H. Wagner, C. Rau, I. K. Robinson, A. Bravin, and A. Olivo, Single-image phase retrieval using an edge illumination $\mathrm{x}$-ray phase-contrast imaging setup, J. Synchrotron Radiat. 22, 1072 (2015).

[27] M. Endrizzi, F. A. Vittoria, G. Kallon, D. Basta, P. C. Diemoz, A. Vincenzi, P. Delogu, R. Bellazzini, and A. Olivo, Achromatic approach to phase-based multi-modal imaging with conventional x-ray sources, Opt. Express 23, 16473 (2015).

[28] M. Chabior, T. Donath, C. David, O. Bunk, M. Schuster, C. Schroer, andF. Pfeiffer, Beam hardening effects in grating-based X-ray phase-contrast imaging, Med. Phys. 38, 1189 (2011).

[29] M. A. Beltran, D. M. Paganin, K. Uesugi, and M. J. Kitchen, $2 \mathrm{D}$ and $3 \mathrm{D} \mathrm{x}$-ray phase retrieval of multi-material objects using a single defocus distance, Opt. Express 18, 6423 (2010).

[30] M. Langer, P. Cloetens, A. Pacureanu, and F. Peyrin, X-ray in-line phase tomography of multimaterial objects, Opt. Lett. 37, 2151 (2012).

[31] L. A. Feldkamp, L. C. Davis, and J. W. Kress, Practical cone-beam algorithm, J. Opt. Soc. Am. A 1, 612 (1984).

[32] R. Bellazzini, G. Spandre, A. Brez, M. Minuti, M. Pinchera, and P. Mozzo, Chromatic X-ray imaging with a fine pitch CdTe sensor coupled to a large area photon counting pixel ASIC, J. Instrum. 8, C02028 (2013).

[33] A. Tapfer, M. Bech, A. Velroyen, J. Meiser, J. Mohr, M. Walter, J. Schulz, B. Pauwels, P. Bruyndonckx, X. Liu, A. Sasov, and F. Pfeiffer, Experimental results from a preclinical x-ray phase-contrast CT scanner, Proc. Natl. Acad. Sci. U.S.A. 109, 15691 (2012).

[34] M. Marschner, M. Willner, G. Potdevin, A. Fehringer, P. B. Noël, F. Pfeiffer, and J. Herzen, Helical X-ray phase-contrast computed tomography without phase stepping, Sci. Rep. 6, 23953 (2016). 